UNSTABLE MAGNETOSONIC WAVES IN A RE:ATIVISTIC PLASMA

\title{
1. LERCHE
}

\section{GPO PRICE \\ s CFSTI PRICE(S) S}

Hard copy $(\mathrm{HC})$

Microfiche (MF)

1,00

f 653 July 65

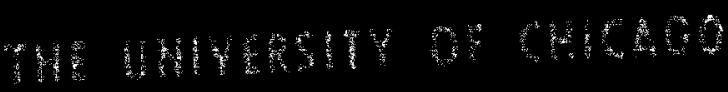

THE PNPICA CFRMI INSTITUTE FOR NUCLAR SUDIES
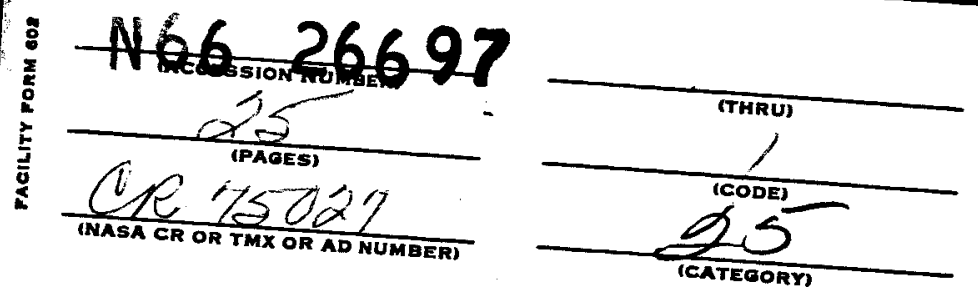


\author{
I. Lerche \\ Enrico Fermi Institute for Nuclear Studies \\ University of Chicago
}

Laboratory for Astrophysics and Space Research

Preprint No. EFINS-66-43

* This work was supported by the National Aeronautics and Space Administration under Grant NASA NsG 96-60. 
Unstable Magnetosonic Waves in a Relativistic Plasma

I. Lerche

Enrico Fermi Institute for Nuclear Studies

University of Chicago

Abstract

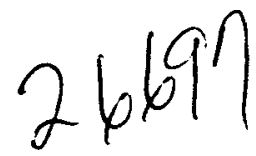

Linearized equations are set up to describe the effect of a circularly

polarized electromagnetic wave on a plasma composed of thermal electrons and protons and also containing an anisotropic relativistic proton component. The unperturbed composite plasma is taken to be embedded in a homogeneous, uniform magnetic field and the wave is taken to propagate along the unperturbed magnetic field.

For wave frequencies very much less than the electron cyclotron frequency it is shown that the relativistic proton plasma will be unstable provided only that $P_{11} \gtrsim P_{\perp}$ where $P_{11}$ and $P_{\perp}$ denote the relativistic plasma pressures parallel and perpendicular to the ambient magnetic field respectively.

The minimum e-folding time is estimated and upon inserting numerical parameters appropriate to the cosmic ray gas in the galactic disc it is shown that parallel anisotropy $\left(P_{11}>P_{\perp}\right)$ will be destroyed in a time short compared to the mean lifetime of cosmic rays in the disc $\left(\sim 6 \times 10^{5}\right.$ years). 
1. Introduction

It is well known that the observed cosmic rays are isotropic to better than $1 \%$ (Greisen, 1956). It is also well known that the average cosmic ray lifetime in the galactic disc is about $6 \times 10^{5}$ years (Parker, 1965). It is therefore of some importance to find mechanisms which will reduce arbitrary degrees of an isotropy to less than the $1 \%$ level in a time short compared to the cosmic ray lifetime. (This is based on the assumption that cosmic rays are produced anisotropically throughout the galactic disc by supernovae, etc.)

It has been conjectured that irregularities in the mean galactic magnetic field are capable of reducing anisotropy through redistribution of pitch angles. However not much is known about the scale size of such irregularities. Consequently it is of interest to examine other possible processes which will reduce anisotropy. In several recent papers (Lerche, 1966a and earlier references therein) we examined the possibility that cyclotron and space charge waves were plausible mechanisms for producing a reduction in anisotropy of the cosmic ray gas. It was found that these waves were indeed capable of producing such a reduction in a reasonable time provided only that $P_{\perp}>P_{11}$.

Since it is not known whether the $1 \%$ anisotropy refers to perpendicular $\left(P_{\perp}>P_{11}\right) \quad$ or parallel $\left(P_{11}>P_{\perp}\right)$ anisotropy it is of some considerable interest to see if any waves exist which will yield an unstable situation for $P_{11}>P_{\perp}$

It can be shown (Lerche, 1966b) that a relativistic plasma will be unstable for $P_{\| 1}>P_{\perp}$ provided also that

$$
4 \pi\left(P_{11}-P_{\perp}\right)>H_{0}^{2}
$$


where $H_{0}$ is the magnitude of the ambient magnetic field. This is an extremely difficult condition to meet for the cosmic ray gas in the galactic disc. with present day
parameTers. We will demonstrate in this paper that for wave frequencies very much less than the electron cyclotron frequency it is possible to make the cosmic ray plasma unstable against the magneto-acoustic mode provided only that $P_{11} \gtrsim P_{\perp}$

2. The Dispersion Relation

We choose a Cartesian co-ordinate system so that the galactic magnetic field, which we take to be homogeneous, uniform and of strength $H_{0}$, points in the Z-direction. Thus

$$
H_{0}=H_{0}(0,0,1)
$$

We take each component of the embedded plasma to be collisionless when it follows that the distribution function, $f^{ \pm}$, for protons $(+)$or electrons $(-)$satisfies

$$
\frac{\partial f^{ \pm}}{\partial t}+\frac{c p}{\sqrt{\left(1+p^{2}\right)}} \cdot \frac{\partial f^{ \pm}}{\partial x} \pm \frac{\epsilon}{m_{ \pm} c}\left[\frac{E}{m}+\frac{p \times H}{\sqrt{\left(1+p^{2}\right)}}\right] \cdot \frac{\partial f^{ \pm}}{\partial p}=0 \text {. }
$$

Here $\in(>0)$ is the charge on a proton, $m_{ \pm}$denotes the rest mass of a proton $(+)$ or electron $(-)$ respectively, and the normalized momentum $f$ is defined in terms of the real momentum, $f^{\prime}$, through $m_{ \pm}<f=f^{\prime}$. The speed of light in vacuo is denoted by $C$.

We now choose to perturb the plasma by a right handed circularly polarized electromagnetic wave whose vector potential, $A$, is given by 
(3)

$$
\underline{n}=A_{0}(1, i, 0) \exp i(k z-\omega t) \text {. }
$$

Setting

$$
f^{ \pm}=f_{0}^{ \pm}+f_{1}^{ \pm} \text {and } \underset{m}{H}=\underset{m}{H_{0}}+\nabla \times \underset{m}{A}
$$

where subscript ' 0 ' denotes equilibrium values, we see that the linearized equation for $f_{1}^{ \pm}$can be written

$$
\begin{aligned}
& \frac{\partial f_{1}^{ \pm}}{\partial t}+\frac{c R}{\sqrt{\left(1+p^{2}\right)}} \cdot \frac{\partial f_{1}^{ \pm}}{\partial x} \pm \frac{\epsilon}{m_{ \pm} c}\left[-\frac{1}{c} \frac{\partial A}{\partial t}+\frac{p \times(\nabla \times A)}{\sqrt{n}\left(1+p^{2}\right)}\right] \cdot \frac{\partial f_{0}^{ \pm}}{\partial R} \\
& \quad \pm \frac{\epsilon\left(R \times H_{0}\right)}{m_{ \pm} c \sqrt{\left(1+p^{2}\right)}} \cdot \frac{\partial f_{1}^{ \pm}}{\partial q}=0
\end{aligned}
$$

Setting

$$
f_{1}^{ \pm}(x, t, R)=f_{1}^{ \pm}(f) \exp i(k z-\omega t)
$$

$$
\begin{aligned}
& \text { we see that } f_{1}^{ \pm}(f) \\
& i f_{1}^{ \pm}\left[\frac{c k p_{z}}{\sqrt{\left(1+p^{2}\right)}}-\omega\right] \pm \frac{i \omega \in H_{0}}{m_{ \pm} c^{2}}\left(\frac{\partial f_{0}^{ \pm}}{\partial p_{x}}+i \frac{\partial f_{0}^{ \pm}}{\partial p_{y}}\right) \pm \frac{\epsilon H_{0}}{m_{ \pm} c \sqrt{ }\left(1+p^{3}\right)}\left(\frac{p_{y} \partial f_{y}^{ \pm}}{\partial p_{x}}-p_{x} \frac{\partial f_{1}^{ \pm}}{\partial p_{y}}\right) \\
& \pm \frac{i \epsilon k A_{0}}{m_{ \pm} c \sqrt{ }\left(1+p^{2}\right)}\left[\left(p_{x}+i p_{y}\right) \frac{\partial f_{0}^{ \pm}}{\partial p_{z}}-p_{z}\left(\frac{\partial f_{0}^{ \pm}}{\partial p_{x}}+i \frac{\partial f_{0}^{ \pm}}{\partial p_{y}}\right)\right]=0 .
\end{aligned}
$$


$-4-$

It can easily be seen from (3) that the equilibrium distribution function, $f_{0}^{ \pm}$, satisfies

$$
f_{0}^{t}(p)=f_{0}^{t}\left(p^{2}, p_{z}\right)
$$

provided that $H_{0}$ has no spatial gradients and that the re is no ambient electric field.

In order to solve (7) for $f_{1}^{ \pm}(p)$ it is convenient to transform to cylindrical coordinates in momentum space defined by

$$
p_{x}=p_{1} \cos \varphi_{,} p_{y}=p_{1} \sin \varphi, p_{z}-p_{11} \text {. }
$$

Upon making use of (8) in the form

$$
\frac{\partial f_{0}^{t}}{\partial \varphi}=0
$$

it is a simple matter to show that the solution to (7) is given by

$$
f_{1}^{ \pm}\left(f_{n}\right)= \pm \frac{\epsilon A_{0} e^{i \varphi}\left[\omega V\left(1+p^{2}\right) \frac{\partial f_{0}^{ \pm}}{\partial p_{ \pm}}+c k\left(\beta_{2} \frac{\partial f_{0}^{ \pm}}{\partial p_{1}}-p_{11} \frac{\partial f_{0}^{ \pm}}{\partial p_{2}}\right)\right]}{m_{ \pm} c^{2}\left[\omega V\left(1+p^{2}\right)+\Omega^{ \pm}-c k p_{11}\right]}
$$

where

$$
\Omega^{ \pm}= \pm \in H_{0}(m+c)^{-1} \text {. }
$$


$-5-$

In order to obtain a self-consistent field we must also satisfy

Maxwell's equation

$$
\nabla^{2} Q-c^{-2} \frac{\partial^{2} Q}{\partial t^{2}}=-4 \pi \epsilon \int d^{3} p \frac{p}{\sqrt{\left(1+p^{2}\right)}}\left(f_{1}^{+}-f_{1}^{-}\right)
$$

Upon substitution for $f_{1}^{ \pm}\left(x, t, R_{1}\right)$ in (11) we see that we must demand $k^{2}-\omega^{2} c^{-2}=4 \pi^{2} \varepsilon^{2} c^{-2} \sum_{t,-} m_{ \pm}^{-1} \int_{-\infty}^{\infty} d p_{p_{1}} \int_{0}^{\infty} d p_{2} \frac{p_{t}^{2}}{\sqrt{\left(1+p^{2}\right)}} x$ $\frac{\left[\omega \sqrt{ }\left(1+p^{2}\right) \frac{\partial f_{0}^{ \pm}}{\partial p_{2}}+\operatorname{ck}\left(p_{2} \frac{\partial f_{t}^{t}}{\partial p_{1}}-p_{11} \frac{\partial \delta_{0}^{t}}{\partial p_{1}}\right)\right]}{\left[\omega V\left(1+p^{2}\right)+\Omega^{ \pm}-e k p_{H}\right]}$

Here the sum over $t_{,}-$denotes the fact that we must sum the terms for protons $(+)$ and electrons $(-)$ respectively.

3. The Thermal Equilibrium Distributions

Before further progress can be made with (13) we require some knowledge of the equilibrium distribution functions. Let us bear in mind that we are looking for instability of the cosmic ray proton plasma. In order to exhibit the basic instability (if any) of this component of the plasma, we treat the thermal background of electrons and protons as being completely cold. Thus for the thermal plasma we can write

$$
f_{0}^{ \pm}=\frac{N_{ \pm} \delta\left(p_{+}\right) \delta\left(p_{11}\right)}{2 \pi p_{L}}
$$

Here $N_{ \pm}$are the number densities of the cold protons $(+)$and electrons $(-)$ 
$-6-$

respectively. Also $\delta(\xi)$ is the usual Dirac $\delta$-function. We further assume that the cosmic ray number density is very much smaller than either $\mathrm{N}_{4}$ or $N_{-}$. Thus we can set $N_{+}=N=N$, say, to a very good approxmotion. Also since the cosmic ray plasma is tenuous we assume that it provides a negligible contribution to the real part of the dispersion relation (13). Under this assumption it can easily be shown that (13) becomes

$$
\begin{aligned}
& k^{2}=\omega^{2} c^{-2}-\omega \omega_{\mu}^{2} c^{-2}\left(\omega+\Omega^{+}\right)^{-1}-\omega \omega_{\beta}^{2}=c^{-2}\left(\omega-\left|\Omega^{-1}\right|\right)^{-1} \\
& +i \pi \omega_{\beta}^{2} \beta c^{-2} I_{m} \int_{-\infty}^{\infty} d p_{m} \int_{0}^{\infty} d p_{1} \frac{p_{1}^{2}}{\sqrt{\left(1+p^{2}\right)}} \\
& {\left[\omega v\left(1+p^{2}\right) \frac{\partial F}{\partial p_{2}}+c k\left(p_{1} \frac{\partial F}{\partial p_{1}}-p_{11} \frac{\partial F}{\partial p_{1}}\right)\right]} \\
& {\left[\omega \sqrt{ }\left(1+p^{2}\right)+\Omega^{+}-c k p_{11}\right]}
\end{aligned}
$$

where

$$
\omega_{p E}^{2}=4 \pi N \epsilon^{2} m_{I}^{-1} \text { and } \beta=n_{c k} N^{-1} \text {. }
$$

Here $n_{C h}$ is the number density of cosmic ray protons and we have written $F\left(P^{2}, P_{n}\right) \quad$ for the cosmic ray equilibrium distribution function.

It should be pointed out that $F$ is normalized so that

$$
2 \pi \int_{-x^{2}}^{\infty} d p_{11} \int_{0}^{\infty} d p_{2} p_{1} F\left(p_{2}^{2}, p_{1}\right)=1
$$


$-7-$

4. Stability Considerations

In order to decide whether the system, whose dispersion relation is given by (15), is unstable or not it can be shown (Pentose, 1960) that it is both necessary and sufficient to define $\quad k$ real and positive and to define $i$ to lie initially in the upper half complex plane. In general (15) can be cast in the form

$$
k^{2}=J(\omega)+i k(\omega),
$$

where $J$ and $K$ are real functions of the complex variable

$\omega$ With $\omega=\omega_{k}+i \gamma$ and $|\gamma| \ll\left|\omega_{R}\right|$ we can write

$$
k^{2} \simeq J\left(\omega_{k}, 0\right)
$$

and

$K\left(\omega_{k}, 0\right)+\left.\frac{\partial K\left(\omega_{R}, \gamma\right)}{\partial \gamma}\right|_{\gamma=0} ^{\gamma} \simeq 0$.

Since $k^{2}$ is meromorphic in $\omega$ it follows that $\left.\frac{\partial K\left(\omega_{k}, \gamma\right)}{\partial \gamma}\right|_{\gamma=0}=\left.\frac{\partial J\left(\omega_{R}, \gamma\right)}{\partial \omega_{R}}\right|_{\gamma=0}=\frac{\partial J\left(\omega_{R}, 0\right)}{\partial \omega_{R}}$

Thus 
$-8-$

$$
\gamma=-K\left(\omega_{R}, 0\right) / \frac{\partial J\left(\omega_{R}, 0\right)}{\partial \omega_{R}} .
$$

Hence it is clear that an unstable situation will develop provided $\gamma>0$. For real $\omega$ we see that

$$
J\left(\omega_{k}, 0\right)=k^{2}=\omega_{k}^{2} c^{-2}-\omega_{k} c^{-2} \omega_{k}^{2}\left(\omega_{k}+l^{+}\right)^{-1}-\omega_{k} c^{-2} \omega_{k}^{2}\left(\omega_{k}-1 R_{(23)}\right)^{-1} \text {. }
$$

For $i_{k}<<\left|\Omega^{-}\right| \quad$ it is a simple matter to show that (23) becomes

$$
J\left(\omega_{R}, 0\right)=\omega_{R}^{2} c^{-2}\left(1+\frac{\omega_{R+}^{2}}{\Omega^{+}\left(\omega_{R}+\Omega^{+}\right)}\right),
$$

and in this regime

$$
\frac{\partial J\left(\omega_{R}, 0\right)}{\partial \omega_{R}}=2 \omega_{R} c^{-2}+\frac{\omega_{R} \omega_{R}^{2}\left(\omega_{R}+2 \Omega^{+}\right)}{c^{2} \Omega^{+}\left(\omega_{R}+\Omega^{+}\right)^{2}}>0 .
$$

Thus under the approximation $\quad \omega_{R}<\left|\Omega^{-}\right|$an unstable situation will develop for this mode provided only that

$$
K(4,0)<0
$$

$K\left(\omega_{n}, 0\right)=\pi \omega_{p}^{2} \beta c^{-2} y_{m} \int_{-\infty}^{\infty} d p_{1} \int_{0}^{\infty} d p_{1} \frac{p_{1}^{2}}{v\left(1+p^{2}\right)}$

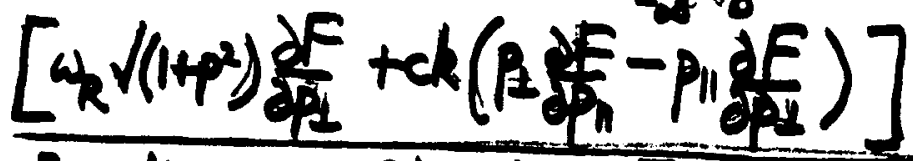

$\left[u_{R} v^{\left(1+p_{2}\right)}+\Omega^{+}-\operatorname{ckp} n\right]$ 
$-9-$

In order to find the imaginary part of the double integral in (27)

for $\omega$ real it is convenient to transform to new variables of integration to avoid the branch cut which appears in the integrand. To this end we let

$$
y=p_{11}, \quad E^{2}=1+p_{2}^{2}+y^{2}
$$

when it follows that

$\frac{\partial F}{\partial P}=y E^{-1} \frac{\partial F}{\partial E}+\frac{\partial F}{\partial y}$

(28a)

$\frac{\partial F}{\partial p_{1}}=\sqrt{ }\left(E^{2}-1-y^{2}\right) E^{-1} \frac{\partial F}{\partial E}$

(28b)

It is then a simple matter to show that (27) becomes

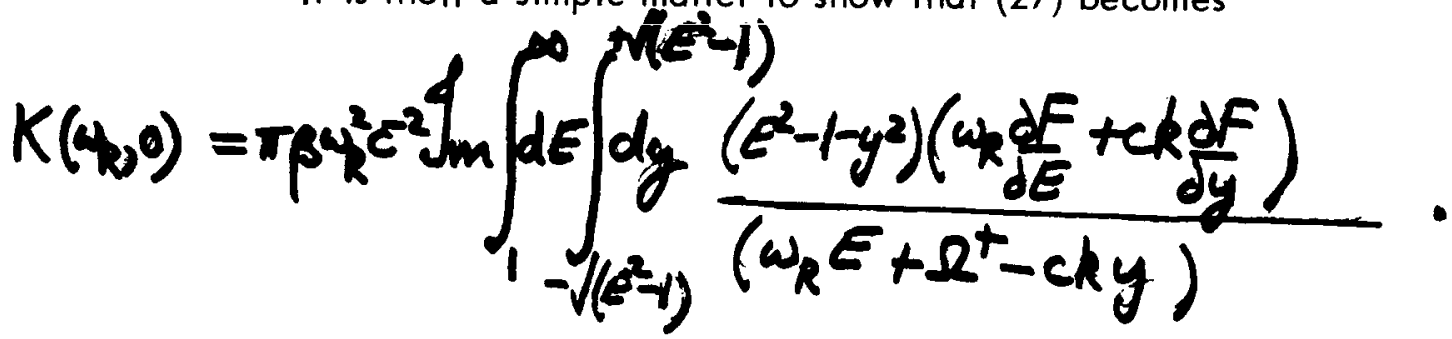

For $R$ real and positive we see that as $\operatorname{Jm}(\omega) \rightarrow 0$ from above we must understand

$$
x^{-1}=P\left(x^{-1}\right)+i \pi \delta(x)
$$

where

$$
X=y-(c k)^{-1}\left(\Omega^{+}+c_{k} E\right)
$$


$-10-$

and we are regarding $x$ as a function of $y$. Here $P\left(x^{-1}\right)$ denotes the principal value of $X^{-1}$.

We see that such an understanding leads to a nonzero value for

$K($ w, 0$)$ provided that

$-c k \sqrt{ }\left(\epsilon^{2}-1\right) \leqslant \Omega^{+}+w_{k} E \leqslant c k \sqrt{ }\left(\epsilon^{2}-1\right)$,

which condition, for a wave travelling in the positive $₹$-direction (ie. $\quad a_{2}>0$ ), demands that

$$
E \geqslant \varepsilon_{0}=\frac{x n^{-1}+\sqrt{\left(1-n^{-2}+x^{2}\right)}}{\left(1-n^{-2}\right)}
$$

where

$$
x=\Omega^{+}(c k)^{-1}=\Omega^{+}(4, n)^{-1}
$$

Here $\boldsymbol{n}$ is the phase refractive index of the wave. It is a simple matter to show that $\quad E>1$ provided $n \geqslant 1$.

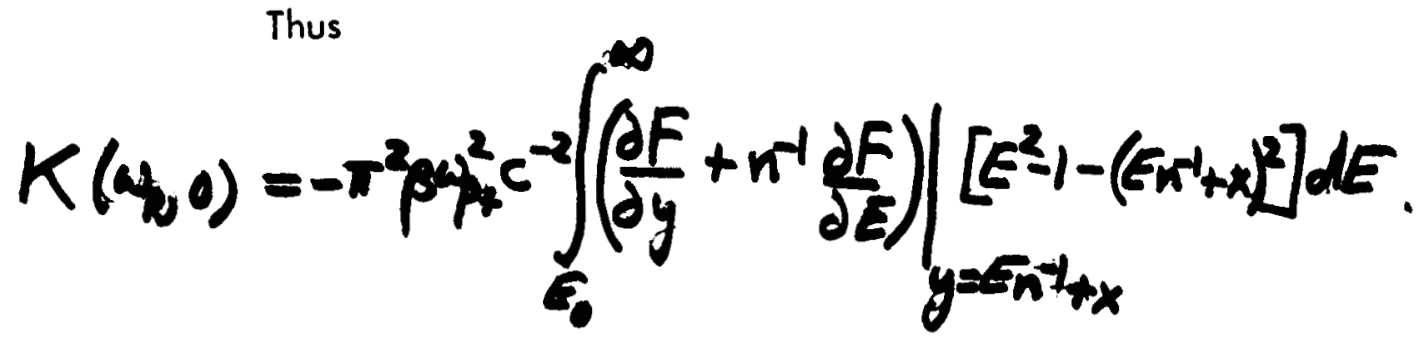

It can be seen by inspection that if $F$ is a monotonically decreasing function of $E$ only, then $K>0$ for all $\omega_{R}$, and 
$-11-$

the system is stable since the requirement for instability is that $K<0$ for some range of

Before further progress can be made with (33) we need to know the cosmic ray equilibrium distribution function. It is well known (Parker, 1965) that at high $E$ values foE $E^{-(2+y)}$ where 2.5583.

In order to demonstrate instability for a part icular cosmic ray distribution function we choose

$$
F(E, y)=S E^{-5}\left(1+\alpha y^{2} E^{-2}\right)
$$

This particular distribution function has the advantage that it enables the integral in (33) to be performed exactly. Also it behaves in a similar manner to the observed cosmic ray spectrum at high $E$ values. At the same time it gives an idea of the response due to the inclusion of a fixed amount of anisotropy through the parameter $\propto$. We do not maintain that (34) represents the observed cosmic ray spectrum but rather that it possesses the essential features of the observed spectrum.

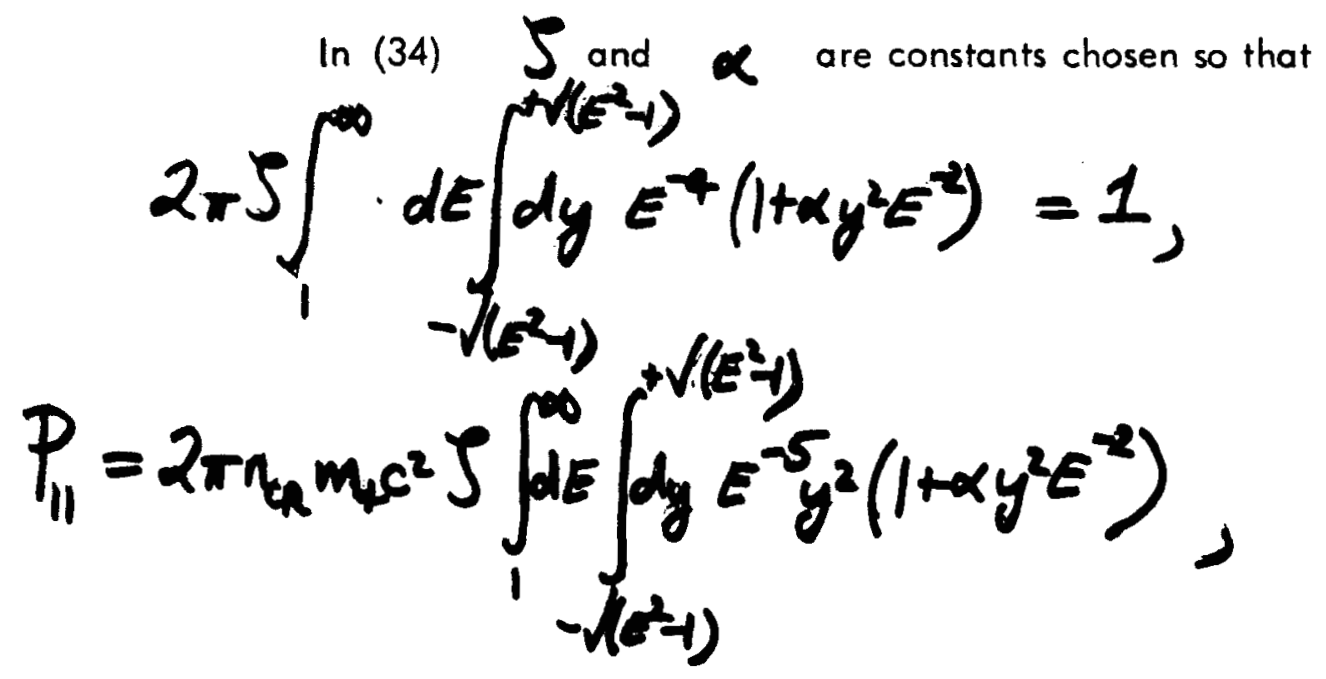




$$
P_{I}=\pi n_{a} m_{+} c^{2} \iint_{1}^{\infty} d \int_{-\sqrt{\left(E^{2}-1\right)}}^{+\sqrt{\left(E^{2}-1\right)}} d y E^{-5}\left(E^{2}+y^{2}\right)\left(1+x y^{2} E^{-2}\right) .
$$

Here $P_{n}$ and $\mathcal{P}_{\mathcal{L}}$ are the cosmic ray gas pressure components parallel and perpendicular to the ambient magnetic field respectively.

In some respects (34) is a rather particular distribution function since it possesses only two independent parameters, namely $S$ and $\alpha$. Thus once the normalization condition and the energy density of cosmic rays are specified the pressure components follow automatically. However this is not a serious failing. The point is that (37) will demonstrate the existence, or otherwise, of a basically unstable situation without involving an extreme amount of non-essential mathematics.

Upon making use of (34) in (33) we see that

$$
\begin{aligned}
& K\left(\omega_{k}, 0\right)=-\pi^{2} \beta \omega_{*}^{2} 5 E_{0}^{-7} x \\
& \left\{\begin{array}{l}
\frac{1}{3} E_{0}^{4} n^{-1}\left(1-n^{-2}\right)\left(2 x^{2}-5-7 x n^{-2}\right)+\frac{x}{2} E^{3}\left[\alpha\left(1-n^{-2}\right)\left(1-7 n^{-2}\right)-n^{-2}\left(2 x^{-5}-7 x n^{-2}\right)\right] \\
-\frac{1}{5} E_{0}^{2} n^{-1}\left[\left(1+x^{2}\right)\left(2 \alpha-5-7 x n^{-2}\right)+4 \alpha x^{2}\left(1-7 n^{-2}\right)-7 x x^{2}\left(1-n^{-2}\right)\right] \\
+\frac{1}{3} E_{0} \alpha x\left[7 x^{2} n^{-2}-\left(1+x^{2}\right)\left(1-7 n^{-2}\right)\right]+\alpha x^{2} n^{-1}\left(1+x^{2}\right)
\end{array}\right\} .
\end{aligned}
$$

Now under the approximation $\left|w_{R}\right|<C\left|\Omega^{-}\right|$

$$
n^{2}=1+\frac{\omega^{2}}{a^{+}\left(\omega^{2}+\Omega^{+}\right)}
$$


$-13-$

so that to find the range of $\omega_{R}$ for which $K<0$ is extremely difficult in general.

We will see later that in the case of the galactic medium $n^{2}>1$ provided $\omega_{R} \ll\left|\Omega^{-}\right|$. Under this approximation there are two limiting cases in which (37) becomes tractable.

(i) $\gg x^{2} \gg n^{-2}$, ie. $\omega_{2} \gg \Omega^{t} n^{-1} \gg \omega_{n} n^{-1}$.

In this case $\Sigma_{0} \geq 1$ and (37) becomes

$$
\begin{aligned}
& K \simeq \frac{-\pi^{2} \beta \omega_{\beta^{2}}^{2} c^{2} \varphi_{\alpha} \Omega^{+}}{6 n n \omega_{k}} \\
& \text { provided } \alpha{ }_{\alpha} O\left(n^{-1}\right) .
\end{aligned}
$$

It is clear that this situation yields an unstable situation $(K<0)$ provided only that $\quad \alpha>0$. Now from (36) it can be shown that

$$
\alpha=\frac{20\left(P_{11}-P_{L}\right)}{3 \pi^{2} m_{1} c^{2} n_{c R}}
$$

Thus an unstable situation develops provided $0.31 y$ that

$$
P_{11}>P_{1} \text { and } \alpha \approx O(n-1) \text {. }
$$

In this case the e-folding time is given by

$$
\tau=\gamma^{-1}=\frac{6 \omega_{R}^{2} m_{+} c^{2} N\left(R_{+}^{2}+w_{k}^{2}\right)^{3 / 2}}{5 \omega_{*}^{2} R_{+}^{4}\left(P_{11}-P_{+}\right)}
$$


$-14-$

It would appear from (42) that the minimum e-folding time occurs as $\omega_{R} \rightarrow 0$. However we are restricted to values of $\omega_{R}$ such that $\omega_{R} \gg \Omega_{+}^{2}\left(\omega_{t}^{2}+\Omega_{+}^{2}\right)^{-1 / 2}$. We therefore turn our attention to the other extreme situation.

(ii) $x^{2}>>1, x^{2}>>n^{-2}$. ie. $\omega_{R} \ll<\ell^{+} n^{-1}, n \gg>1$.

In this case $E_{0} \simeq x$ and (37) becomes

$$
\begin{gathered}
K \cong-\frac{\pi^{2}}{6} \beta \omega_{*}^{2}+3 \omega_{4}^{3} \Omega_{+}^{-6}\left(\Omega_{+}^{2}+\omega_{*}^{2}\right)^{3 / 2} \\
\text { provided } \quad \alpha \geqslant O(n-1) .
\end{gathered}
$$

In this case we see that an unstable situation will develop provided

$$
P_{11}>P_{\perp} \text { and } \alpha \approx O\left(n^{-1}\right) \text {. }
$$

The e-folding time in this case is given by

$$
\tau=\gamma^{-1}=\frac{3 m_{+} c^{2} N \Omega_{4}^{4}}{5 \omega_{p_{4}}^{2} w_{4}^{2} \sqrt{\left(w_{4}^{2}+\Omega_{4}^{2}\right)\left(P_{11}-P_{1}\right)}}
$$

This e-folding time increases without limit as $\omega_{R} \rightarrow 0$. Thus from (42) and. (45) we see that $\tau$ possesses a minimum value when

$$
w_{k} \simeq \frac{2^{-1 / 4} \Omega_{t}^{2}}{\sqrt{\left(\omega_{t}^{2}+\Omega_{t}^{2}\right)}}
$$


$-15-$

and at this value of $\omega_{R}$

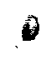

-

This result is derived under the approximations

$\omega_{R} \ll \mid \Omega^{-1}, \frac{\omega_{p_{*}^{2}}^{2}}{\Omega^{2}\left(\omega_{k}+\Omega^{2}\right)}+1 \gg 1, P_{11}-P_{1} \approx O\left(\frac{3 \beta H_{1}^{2}}{320}\right)$.

We will see later that these conditions are easily realized in the case of the galactic plasma.

The wavelength of this most unstable wave is given by

$$
\lambda=2^{5 / 4} \pi \subset \Omega_{+}^{-1} J\left(1+\Omega_{4}^{2} \omega_{p}^{-2}\right)
$$

5. Numerical Estimates

In order to decide whether the instability proceeds at a significant rate for the galactic plasma we need to know $N, H_{0}, n_{C_{R}}$ and

$$
P_{n}-P_{L}
$$

It is known from observations that $H_{0} \simeq 5 \times 10^{-6} \Gamma$ (Davies, 1965).

The number density of cosmic ray protons has been estimated (Parker, 1965) to be about $10^{-10} \mathrm{~cm}^{-3}$. For $\propto$ sufficiently small compared to unity, but positive, we can write $P_{11}-P_{1}=0 \operatorname{2} \times P_{0}$ where $P_{0}$ is the mean cosmic ray pressure which is estimated to be about $3 \times 10^{-13}$ dynes $/ \mathrm{cm}^{2}$. 
$-16-$

Inserting these numbers we see that (47) becomes

$\tau_{\min } \simeq 15 \times N^{1 / 2} \alpha^{-1}$ years.

$\hat{\imath}$

Even for the unlikely case where the number of thermal protons per unit volume is the same as the neutral hydrogen number density $\left(\sim 1\right.$ atom $\left.\mathrm{cm}^{-3}\right)$ we see that $\tau_{\min } \lesssim 6.10^{5}$ years for $\propto \geq 2.5 \times 10^{-5}$.

In this case we find the associated frequency, $f\left(=\omega_{2} / 2+1\right)$ and wavelength are given by

$f \simeq 5 \times 10^{-7} \mathrm{cs}$

(Fla)

and

$\lambda \simeq 3 \times 10^{12} \mathrm{~cm}$.

respectively.

The phase velocity of this wave is

wok

and is given

by

$\omega_{n} / k \simeq 15 \mathrm{~km} \cdot \mathrm{sec}^{-1}$.

For the thermal protons the resonant velocity is

$\omega / k+e^{+} / k=v_{11}$

which, in the present circumstances, is about $4.10^{10} \mathrm{~cm} . \mathrm{sec} .^{-1}$. This velocity, which 
is greater than the velocity of light in vacuo, is in reality so far out on the tail of the thermal distribution that the thermal particles 'resonance behavior can be neglected. Thus we are justified, a posteriori, in treating the thermal gas as completely cold.

In order that the results have some meaning we must satisfy the inequalities (48). These can be written

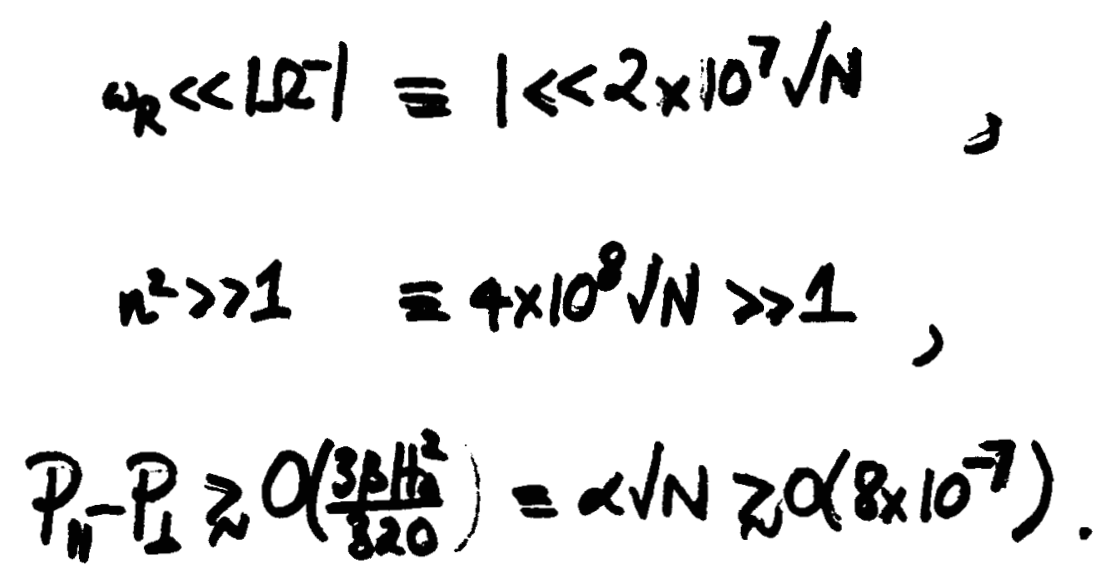

It is clear that we can satisfy (53) for reasonable values of $\mathbf{N}$ and still obtain 2-folding times which are short compared to the mean cosmic ray lifetime in the galactic disc since $\tau_{\min } \lesssim 6.10^{5}$ years only demands that

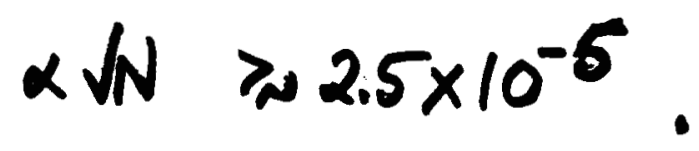

\section{Conclusion}

We have shown that, for reasonable values of the relevant parameters, the an isotropic cosmic ray gas is unstable to the right handed circularly polarized wave with an e-folding time very much less than the mean cosmic ray lifetime in the galactic disc. This result is valid provided 


\section{$4 \pi H_{0}^{-2}\left(P_{11}-P\right) \geqslant O\left(\frac{n_{n}}{10 N}\right)$}

This condition can easily be met for the cosmic ray gas with present day parameters.

We conclude that this mechanism is an extremely powerful one for

reducing parallel anisotropy to less than the $1 \%$ level in a time short compared to the mean cosmic ray lifetime.

This work was supported by the National Aeronautics and Space Administration under Grant NASA NsG-96-60. 
References

Davies, R. D., 1965, Ninth International Conference on Cosmic Rays, London, September.

Greisen, K., 1956, "Progress in Cosmic Ray Physics," Volume 3, Chapter 1, North Holland Publishing Co., Amsterdam.

Lerche, I., 1966a, University of Chicago Technical Report EFINS-66-05 (submitted to Phys. Fluids, February).

Lerche, I., 1966b, Astrophys. J., September (to be published).

Parker, E. N., 1965, "Nebulae and Interstellar Matter," Vol. 7, Chap. 16, ed. Aller, McLaughlin, Middlehurst (to be published).

Penrose, O., 1960, Phys. Fluids, 3, 196. 